\title{
Cation Exchange Strategy to Construct a Targeting Nanoprobe for Enhanced Positive MR Imaging Capability
}

\section{Siyu Chen}

Southern Medical University

Haitao Sun

Zhongshan Hospital Fudan University

\section{Yingyan Zheng}

Huashan Hospital Fudan University

\section{Qian Chen}

Shanghai Institute of Ceramics Chinese Academy of Sciences

Yu Luo ( $\nabla$ yuluo@tongji.edu.cn )

Tongji University https://orcid.org/0000-0002-7088-0715

\section{Hangrong Chen}

Shanghai Institute of Ceramics Chinese Academy of Sciences

\section{Quan Zhou}

Southern Medical University

\section{Research}

Keywords:

Posted Date: June 4th, 2020

DOl: https://doi.org/10.21203/rs.3.rs-32004/v1

License: (9) This work is licensed under a Creative Commons Attribution 4.0 International License. Read Full License 


\section{Abstract}

Background: Excellent imaging performance and good biocompatibility of contrast agents are considered as prerequisites for accurate tumor diagnosis and treatment.

Results: Herein, a novel imaging nanoprobe with actively targeting performance based on ultrasmall paramagnetic iron oxide (USPIO) was constructed by a facile cation exchange strategy followed by conjugation with transferrin (Tf). The stable gadolinium $\left(\mathrm{Gd}^{3+}\right)$ chelation endows the nanoparticles (NPs) with a low value of $r_{2} / r_{1}$ (1.28) and relatively high $r_{1}$ value of $3.2 \mathrm{mM}^{-1} \mathrm{~s}^{-1}$, enabling their use in $T_{1}$ weighted positive MR imaging.

Conclusion: This constructed transferrin modified gadolinium-iron chelate nanoprobe, named as TUG, shows high biocompatibility within a given dose range. More importantly, compared with clinically used Gd-based small molecule contrast agents, the obtained TUG can be more engulfed by breast cancer cells, showing much enhanced $T_{1}$-weighted positive MR imaging in either subcutaneous or in situ tumor models of breast cancer. This novel nanoprobe holds enormous promise to be utilized as a targeting contrast agent with high efficacy for $\mathrm{T}_{1}$-weighted positive MR imaging.

\section{Background}

Magnetic resonance imaging (MRI) has become one of the most common clinical examination methods due to its good tomographic capabilities and high contrast of soft tissue ${ }^{1-4}$. But the sensitivity and efficiency of cancer diagnoses have generally been impeded by the dilemma in identifying tumor tissue from normal tissue ${ }^{5-7}$. To overcome the above obstacles, gadolinium (Gd)-based small molecule chelates are currently used in clinical practices as contrast agents to improve the contrast ${ }^{8-11}$. However, these gadolinium-based $T_{1}$ contrast agents contain free toxic gadolinium, which potentially leads to renal fibrosis and other toxicity concerns, and these agents still have some other shortcomings, such as short blood circulation time, no target specificity, etc ${ }^{12,13}$. To reduce the side effects of gadolinium-based contrast agents, an iron-based contrast agent is becoming a promising alternative. Although those contrast agents with iron oxide nanoparticles (NPs) as the cores have higher biocompatibility compared to gadolinium-based contrast agents ${ }^{14,15}$, most iron-based contrast agents are negative contrast agents that lead to insurmountable disadvantages, such as difficulty in distinguishing from bleeding, metal deposits, or calcification ${ }^{16-18}$. Therefore, the construction of a positive contrast agent based on iron species to reduce the content of gadolinium ion and to improve the biocompatibility of the contrast agent is highly demanded ${ }^{19}$.

With the decrease of the size of iron oxide NPs, the specific surface area of NPs increases and the energy exchange of $T_{1}$ becomes faster ${ }^{20,21}$. The tissue signal collected from $T_{1}$-weighted image increases and $\mathrm{T}_{2}$ effect decreases, which can be used for $\mathrm{T}_{1}$-weighted MRI ${ }^{17,22,23}$ When the size of ultra-small paramagnetic iron oxide (USPIO) NPs is less than $5 \mathrm{~nm}$, the performance of negative contrast agents will 
be greatly weakened, while the performance of positive contrast agents will be greatly improved 24,25 . However, practically the $T_{1}$-weighted MR imaging performance of USPIO is not super to that of clinically used gadolinium-based small molecule contrast agents ${ }^{26,27}$ due to seven unpaired electrons of gadolinium. Herein, to improve the $T_{1}$-weighted MRI performance of USPIO, a facile cation exchange strategy was adopted to solvate the carboxyl groups covering ultra-small paramagnetic iron oxide NPs prepared by a solvothermal method with $\mathrm{Gd}(\mathrm{III})$ ions. Compared with the gadolinium-based contrast agent, Gd(III) ion-induced self-assembled NPs, named as USPIO@Gd(III) (Abbr. UG), present good biocompatibility and stability with tunable gadolinium content.

It is well known that small-molecule contrast agents currently used in clinical practice do not have the tumor-targeting ability. To increase the accumulation of contrast agents in tumor site, we use chemical crosslink to attach transferrin on the surface of UG. Due to the high expression of transferrin receptors in tumor cell membranes ${ }^{28}$, the binding of transferrin to receptors can increase the number of nanoparticles entering the tumor cells. The prepared UG was thus further modified with transferrin to form the targeted imaging nanoprobe, namely TUG, which shows good colloidal stability, high $r_{1}$ relaxivity, and good biocompatibility. More importantly, TUG shows enhanced cellular uptake efficacy by breast cancer cells, indicating good tumor-targeting imaging and longer MRI window time performance.

\section{Results And Discussion}

\subsection{Preparation and Characterization of TUG}

A one-step solvothermal method is proposed to prepare ultrasmall paramagnetic iron oxide with stable surface modification of sodium citrate (Fig. 1). Due to a large amount of sodium citrate on the surface of nanoparticles, USPIO has a strong charge repulsion force, which is beneficial to maintain excellent colloidal stability in different liquid environments ${ }^{29}, 30$. The UG NPs were obtained by the cation exchange between $\mathrm{Gd}(\mathrm{III})$ and sodium citrate on the surface of USPIO. Subsequently, carboxyl groups covered the nanoparticles and amino groups on the transferrin were bonded via an EDC/NHS reaction ${ }^{31,32}$, resulting in the final product TUG.

The photograph of TEM reveals that the prepared ultrasmall iron oxide nanoparticles are uniformly dispersed with a stable diameter of about $2 \mathrm{~nm}$ (Fig. 2a). After addition of Gd ions and Tf, the obtained TUG formed by the assembly of ultrasmall iron oxide shows good dispersion with a diameter of about $50 \mathrm{~nm}$ (Fig. 2b). SEM element line scanning analysis of TUG clearly shows three elements of iron, gadolinium, and oxygen homogeneously co-present in the obtained nanoprobe (Fig. S1). Figure 2c shows the zeta potentials and hydrodynamic sizes of the USPIO, UG, and TUG. In detail, the zeta potential of USPIO is $-40.0 \mathrm{mV}$ due to the abundant carboxyl groups, while after addition of Gd(III) and Tf, the zeta potential increased to $-20.0 \mathrm{mV}$ and $-18.0 \mathrm{mV}$, respectively, indicating successful surface modification (Fig. 2c). The hydrodynamic sizes of USPIO, UG, and TUG are $2 \mathrm{~nm}, 50 \mathrm{~nm}$, and $82 \mathrm{~nm}$, respectively, which also implying the formation of nanoprobe with successful surface modification of transferrin. 
Furthermore, the results of zeta potentials and hydrodynamic sizes of TUG in different media $\left(\mathrm{H}_{2} \mathrm{O}, \mathrm{PBS}\right.$, and RPMI) for 7 days indicate that TUG can maintain colloidal stability for a long time, which is suitable to in vivo application (Fig. S2). During the synthesis process, the feed ratio of Fe/Gd was set to 10:1, and the actual ratio of Fe/Gd in TUG measured by ICP-OES was 16.7:1. Additionally, ICP-OES results show that the molar ratio of Fe/ Na in USPIO nanoparticles and TUG is 3.7 and 61.3, respectively, confirming that sodium ions have been successfully exchanged by Gd ions (Fig. 2d). The conjugation of transferrin was confirmed by FTIR and TGA (Fig. $2 \mathrm{e}$ and Fig. 2f). After modification with transferrin, the absorption peaks at $3390 \mathrm{~cm}^{-1}, 2930 \mathrm{~cm}^{-1}, 1652 \mathrm{~cm}^{-1}$, and $584 \mathrm{~cm}^{-1}$ correspond to the $\mathrm{O}-\mathrm{H}, \mathrm{C}-\mathrm{H}, \mathrm{N}-\mathrm{H}$, and Fe-O stretching of TUG, indicating the successful modification of Tf. The TGA curve (Fig. 2f) shows a modest decline at the beginning of the test implying a $10 \%$ weight loss before $100^{\circ} \mathrm{C}$ caused by the adsorbed water in TUG. The total weight loss from $200{ }^{\circ} \mathrm{C}$ to $500^{\circ} \mathrm{C}$ due to the thermal decomposition of organics is around $55 \%$, in which the amount of Tf can be calculated to be $37.5 \%$. Additionally, the stability experiment of TUG verifies that less than $5 \%$ free gadolinium ions released from TUG after 8 hours in the saline (Fig. 2g), which ensures its hypotoxicity.

\section{2 $\mathrm{T}_{1}$ Relaxometry and MRI Imaging}

The $r_{2}$ and $r_{1}$ values of USPIO and TUG can be calculated by plotting the reciprocal of relaxation time $\left(1 / \mathrm{T}_{2}\right.$ or $\left.1 / \mathrm{T}_{1}\right)$ as a function of Fe concentration (Fig. $2 \mathrm{~h}$ and Fig. $\left.2 \mathrm{i}\right)$. It is found that, for USPIO, the $\mathrm{r}_{1}$ and $r_{2}$ values are $0.1 \mathrm{mM}^{-1} \mathrm{~s}^{-1}$ and $0.6 \mathrm{mM}^{-1} \mathrm{~s}^{-1}$, respectively, with the $r_{2} / r_{1}$ ratio of 6.0 . Interesting, for TUG, the $r_{1}$ and $r_{2}$ values are $3.2 \mathrm{mM}^{-1} \mathrm{~s}^{-1}$ and $4.1 \mathrm{mM}^{-1} \mathrm{~s}^{-1}$, respectively, with the $r_{2} / r_{1}$ ratio of 1.3 . Compared with USPIO, TUG shows higher $r_{1}$ and lower ratio of $r_{2} / r_{1}$, which could be ascribed to the shortened longitudinal relaxation time due to the synergistic effect of the obtained cluster structure.

\subsection{Phagocytosis, Cytotoxicity and in vitro MRI of TUG in $4 \mathrm{~T} 1$ cells}

The cytocompatibility of the contrast agents is the premise of applying TUG in vivo. As shown in Fig. 3a, after $24 \mathrm{~h}$ co-culture, TUG shows negligible cytotoxicity to $4 \mathrm{~T} 1$ cells (the cell viability of $4 \mathrm{~T} 1$ cells is above $90 \%$ within the given concentration from $0-100 \mu \mathrm{g} / \mathrm{mL}$ ). It is worth mentioning that when the coincubation time prolonged to $48 \mathrm{~h}$ under iron content of $100 \mu \mathrm{g} / \mathrm{mL}$, the cell survival rate remained above $80 \%$, indicating good cytocompatibility of prepared TUG. Furthermore, we validated TUG MRI performance at the cellular level before performing in vivo animal imaging. As shown in Fig. 3b-c, regardless of TUG group or commercial contrast agent Magnevist (Gd-DTPA), the MR signal of 4T1 cells after co-incubation increases with increasing concentration of different contrast agents. Interestingly, at the same concentration, the MR signal of the TUG group is remarkably higher than that of the commercial contrast agent group, indicating that TUG has better imaging performance than the commercial contrast agent. Besides, the introduction of transferrin increases the cell

phagocytosis of nanoparticles due to the overexpressed transferrin receptors of 4T1 breast cancer cells. We thus evaluated the difference in phagocytosis of TUG and UG by ICP-OES and Prussian blue staining. 
As shown in Fig. 3e, at the same dose, the phagocytosis of TUG by 4T1 cells reaches $36.1 \mu \mathrm{g}$ per 100,000 cells, while the phagocytosis of non-targeted nanoparticles is only $8.3 \mu \mathrm{g}$ per 100,000 cells. The amount of nanoparticles consumed by cells in TUG group was 4.3 times of the UG group and the statistical results proved that the modification of transferrin considerably increases the phagocytosis of cells by nanoclusters. The results of Prussian blue staining show that 4T1 cells treated with TUG show clear blue color, while almost no blue signals appeared in the saline-combined UG group, which was consistent with the ICP results (Fig. 3d-e).

\subsection{MRI of TUG Nanoprobes in vivo}

The enhancement effect of TUG as an MRI contrast agent in vitro was studied by comparing and analyzing $T_{1}$-weighted MRI images (Fig. S3). The MRI of TUG shows that $T_{1}$ signal increases with the increase of the Gd proportion. Then, MR images of mice were obtained for further study of the

enhancement effect of MRI (Fig. 4 and Fig. S6). T $_{1}$-weighted MR images of whole tumor areas were acquired before and $1 \mathrm{~h}$ and $8 \mathrm{~h}$ after injection by recording the tumor area signal values from all periods and drawing average luminance histogram of MR image tumor area. In the subcutaneous tumor model, the signal peak was detected in mice injected with Magnevist $10 \mathrm{~min}$ after injection, since Magnevist only passively accumulates in the peripheral vascular tissues of tumor sites by EPR effect, resulting in a low concentration of TUG in the tumor region, and almost unchanged relaxation time of regional hydrogen proton $T_{1}$. Eight hours after the injection of TUG, the MR images of the tumor areas of the mice are not only brighter than those before the injection, but also brighter than those during the peak in the tumor areas of the mice injected with Magnevist. In the tumor models in situ, Magnevist's signal peak time is about 30 min, and the magnitude of Magnevist's signal peak occurs in less than 0.5 hours after the injection of TUG. It is proved that the $T_{1}$-weighted imaging effect of TUG is superior to Magnevist in tumor regions. Notably, the peak time varies in different tumor models, which could be ascribed to the fact that the blood supply of subcutaneous tumor is not as rich as that of breast tumors in situ, leading to longer peak time.

\subsection{Safety Evaluation of TUG}

The long-term biosafety of TUG in living mice after intravenous injection was also evaluated. Section pictures of organs stained by Hematoxylin and eosin (H\&E) showed that physiological morphologies of organs including heart, lung, liver, spleen, and kidney of Balb/c female mice remained normal for 14 days after injection of TUG even at a high dose of $15 \mathrm{mg} / \mathrm{kg}$ (Fig. 5). To determine whether TUG hurts liver function and renal function, the levels of enzymes and chemicals in serum such as alanine aminotransferase (ALT), aspartate aminotransferase (AST), blood urea nitrogen (BUN) and creatinine (CRE) were measured and no abnormals found. Also, the hematocrit, hemoglobin, mean corpuscular volume, mean corpuscular hemoglobin, lymph cells, platelet, red blood cells, red cell distribution, and white blood cells remained almost unchanged after injection of TUG. All the above results indicate high biosafety of the prepared TUG. 


\section{Experimental \\ 3.1 Materials}

Ferric chloride $\left(\mathrm{FeCl}_{3} \cdot 6 \mathrm{H}_{2} \mathrm{O}\right)$ was offered by Sinopharm Chemical Reagent Co., Ltd. Diethylene glycol (DEG), sodium citrate was acquired from Sigma-Aldrich (Shanghai, China). AnhydrousSodium acetate ( $\mathrm{NaOAc}$ ) and gadolinium chloride hexahydrate were received from Shanghai Macklin Biochemical Co., Ltd. Transferrin was purchased from Shanghai Aicheng Biotechnology Co., Ltd. 1-[3-(dimethylamino) propyl]-3-ethylcarbodiimide hydrochloride (EDC), and N-hydroxysuccinimide (NHS) were acquired from ROTRN Biotechnology Co., Ltd. 4T1 cells were obtained from Institute of Biochemistry and Cell Biology (Chinese Academy of Sciences,

Shanghai, China) and BALB/C nude mice of SPF were offered by Shanghai SLAC Laboratory Animal Co., Ltd. CCK-8 kit and Prussian blue dye were all purchased from Beijing Leagene Biotechnology Co. Ltd. Fetal bovine serum and other reagents used for cell culture were purchased from Gibco Co., Ltd.

\subsection{Synthesis of TUG Nanoprobe}

Sodium citrate stabilized USPIO was prepared according to our previous reported literature ${ }^{33}$. Then, under magnetic stirring at $0{ }^{\circ} \mathrm{C}$ the gadolinium chloride aqueous solution $(5.5 \mathrm{~mL}, 2 \mathrm{mg} / \mathrm{mL})$ was dropwise added into USPIO aqueous solution ( $10 \mathrm{~mL} 5 \mathrm{mg} / \mathrm{mL}$ ). After 2 hours of reaction, the obtained USPIO@Gd(III) NPs (Abbr. UG) were dialyzed against deionized water (6 times, 2 L) for 2 days in a dialysis membrane (MWCO 8,000 Da). Subsequently, Tf was combined with UG as a whole through the

reaction between $-\mathrm{NH}_{2}$ and $-\mathrm{COOH}$ in the presence of $\mathrm{EDC} / \mathrm{NHS}$. According to the instructions of the reagents, $1 \mathrm{~mL}$ EDC $(13 \mu \mathrm{mol})$ and $1 \mathrm{~mL} \mathrm{NHS}(13 \mu \mathrm{mol})$ were added into $5.6 \mathrm{~mL}$ of UG (ice bath precooling) under magnetic stirring for 1.5 hours. After that, $2.4 \mathrm{~mL}$ of $\mathrm{Tf}(17 \mathrm{mg} / \mathrm{mL})$ was dropwise added into UG/EDC/NHS mixtures. After $5 \mathrm{~h}$ magnetic agitation reaction at room temperature, TUG nanoprobe was purified by membrane dialysis (MWCO 8,000 Da) to remove the unreacted free EDC and NHS. The final products were named as TUG.

\subsection{Characterization of TUG}

The morphology of USPIO and UG were characterized by scanning electron microscopy (SEM) and transmission electron microscopy (TEM). Cation exchange between sodium and gadolinium ions was characterized by Inductively Coupled Plasma Optical Emission Spectrometer (ICP-OES). Size distribution, zeta potentials, Fourier transform infrared (FTIR), thermogravimetry (TGA), and MR imaging were conducted to characterize the nanoclusters.

\subsection{Colloidal Stability and Gd(III) Release Test}

The colloidal stability of nanoparticles is a crucial index to evaluate the possibility of their subsequent biological applications. We estimated the colloidal stability of the prepared nanoparticles by monitoring the hydrated particle size changes of TUG dispersed in deionized water, PBS, and serum-containing 
medium for 7 days. The release of $\mathrm{Gd}(\mathrm{III})$ from chelating agents is one of the potential toxic and adverse effects of clinically used Gd(III)-based contrast agents. Therefore, we tested the cumulative release of $\mathrm{Gd}$ (III) from the dialysis bag to determine the stability of the nanocluster prepared by the cation exchange strategy. Briefly, $3 \mathrm{~mL}$ TUG solution (Fe concentration $10 \mathrm{~g} / \mathrm{mL}$ ) was added into a dialysis bag (MWCO $8,000 \mathrm{Da}$ ), and the dialysis bag was then placed in a $50 \mathrm{~mL}$ centrifuge tube containing $20 \mathrm{~mL}$ of saline and shaken in a constant temperature shaker $\left(37^{\circ} \mathrm{C}, 120 \mathrm{rpm}\right) .5 \mathrm{~mL}$ of external liquid in the tube was collected at a settled time ( $15 \mathrm{~min}, 30 \mathrm{~min}, 1 \mathrm{~h}, 2 \mathrm{~h}, 4 \mathrm{~h}$, and $8 \mathrm{~h}$ ) and $5 \mathrm{~mL}$ of new saline was replenished. All samples were then tested by ICP-OES for Gd(III) measurement.

\subsection{Toxicity Analysis and Cellular Uptake}

CCK8 method was selected to study the cytotoxicity of TUG to 4T1 cells (mouse breast cancer cells). 4T1 cells were seeded at a cell density of $1 \times 10^{4}$ per well in 96 -well plate containing $0.2 \mathrm{~mL}$ of RPMI medium, and incubated at $37{ }^{\circ} \mathrm{C}$ in a humidified atmosphere with $5 \% \mathrm{CO}_{2}$ for $12 \mathrm{~h}$. Next, the nutrient-depleted medium was replaced by fresh medium containing TUG of different concentrations at $37^{\circ} \mathrm{C}, 5 \% \mathrm{CO}_{2}$ for $12 \mathrm{~h}$ and $24 \mathrm{~h}$. And then the culture medium was replaced by fresh serum-free medium $(90 \mu \mathrm{L})$ containing CCK8 $(10 \mu \mathrm{L}, 10 \%)$ for $4 \mathrm{~h}$ incubation. Absorbance at $450 \mathrm{~nm}$ in each well was measured by Bio-Tek ELX800 spectrophotometric microplate reader (Vermont, America), based on which cell viability was calculated. All experiments were conducted three times in parallel. The phagocytosis of UG and TUG by 4T1 cells was evaluated by using ICP-OES and Prussian blue staining. Briefly, 4T1 cells were seeded in a 6-well plate at a density of $5 \times 10^{5}$ cells/well for $16 \mathrm{~h}$ Then the culture medium was replaced by fresh medium containing UG or TUG in each well for $4 \mathrm{~h}$ incubation. The cells were washed three times with PBS before the Prussian blue staining and ICP-OES test.

\subsection{In vitro $\mathrm{T}_{1}$-weighted MR Imaging Performance of TUG}

4T1 cells were cultured in the same environment as mentioned above for $8 \mathrm{~h}$ and then the culture medium was replaced by a fresh medium with or without TUG, or UG $\left(\mathrm{C}_{\mathrm{Gd}}\right.$ 0.1-0.00125 mM). After the subsequent $2 \mathrm{~h}$ treatment, the cells were washed three times by PBS, treated with trypsin for $3 \mathrm{~min}$, and then collected by centrifuge at $1000 \mathrm{rpm}$ for $5 \mathrm{~min}$. The obtained cells were digested with Aqua regia for Gd measurement by ICP-OES. For MRI, the obtained cells were dispersed in $0.5 \mathrm{~mL}$ of agarose $(0.5 \%)$ for MR scanning using a $3.0 \mathrm{~T}$ clinical MR imaging system under the following parameters: $F O V=60 \times$ $60 \mathrm{~mm}$, matrix $=256 \times 256$, section thickness $=2 \mathrm{~mm}, \mathrm{TR}=1200 \mathrm{~ms}$, and TE $=17 \mathrm{~ms}$.

\subsection{In vivo MR Imaging of Breast Tumor}

Animal experiments were carried out according to protocols approved by the Southern Medical University Committee Animal Care and Use Committee, and also following the policy of the National Ministry of Health. 4 weeks old Balb/c female nude mice (body weight $\approx 18 \mathrm{~g}, \mathrm{n}=12$ ) were purchased from Beijing Vital River Laboratory Animal Technology Co., Ltd, and randomly divided into two groups with six in each group. $3 \times 10^{6} 4 \mathrm{~T} 1$ cells/mice were implanted under the skin of the right-back and mammary gland to establish a subcutaneous tumor model and in situ tumor model of breast cancer. When the breast tumor 
nodules reached a volume of about $200 \mathrm{~mm}^{3}$ after approximately 7 days, each group $(n=6)$ was divided into two subgroups $(n=3)$ and injected with an equal dose of Magenevist and TUG $(1 \mathrm{mg} / \mathrm{mL}, 250 \mu \mathrm{L})$ through the tail vein for later analysis of the signal enhancement in the tumor site. After injection, the mice were anesthetized by intraperitoneal injection of $5 \%$ chloral hydrate $(120 \mu \mathrm{L})$. The $\mathrm{T}_{1}$-weighted images were obtained by a $0.5 \mathrm{~T}$ MRI system at $0 \mathrm{~h}, 2 \mathrm{~h}, 4 \mathrm{~h}, 6 \mathrm{~h}, 8 \mathrm{~h}$. The parameters of MRI in vivo were set as follows: $\mathrm{TR}=360 \mathrm{~ms}, \mathrm{TE}=13 \mathrm{~ms}$, matrix $=256 \times 160$, section thickness $=2 \mathrm{~mm}$, and FOV $=80 \times$ $80 \mathrm{~mm}$. The brightness of the tumor was measured by MATLAB 7.0. The enhancement of brightness showed an increased $\mathrm{T}_{1}$-weighted signal.

\subsection{In vivo Biodistribution and Toxicity Assessment}

After the nanoprobes were delivered to the mice through the tail vein, their toxicity to the mice determines the fate of biomedical applications. The in vivo toxicity of the nanoprobe was analyzed 14 days after injection of different doses of the nanoclusters, and the blood biochemistry, changes in blood routine indexes, and tissue staining of organs were analyzed.

\subsection{Statistical Analysis}

The significant difference between the experimental statistics is analyzed by One-way ANOVA and Tukey's multiple comparison tests. When the P-value $<0.05$, statistics were regarded to be significantly different. $\mathrm{P}<0.05$ was indicated by $(*), P<0.01$ by $(* *)$ and $P<0.001$ by $(* * *)$.

\section{Conclusions}

In summary, a facile cation exchange strategy followed by conjugation with transferrin (Tf) was developed to construct a novel TUG nanoprobe with excellent biocompatibility. The low value of $r_{2} / r_{1}$ (1.28) and relatively high $r_{1}$ value of $3.2 \mathrm{mM}^{-1} \mathrm{~s}^{-1}$ enable the obtained TUG an excellent candidate as $T_{1}{ }^{-}$ weighted MR imaging contrast agent. The in vivo results of either the subcutaneous or in situ tumor models of breast cancer confirm that the TUG has the obviously enhanced $T_{1}$-weighted MR imaging performance to the clinically used Gd-based small molecule contrast agents. Therein, TUG with transferrin modification enabling high specific binding performance to the transferrin receptor of breast cancer also greatly contributes to the high capability of $T_{1}$-weighted MR imaging. Such targeted specific binding nanoprobes are expected to be highly promising in biomedical applications, such as efficient MRI probe for cancer diagnosis.

\section{Declarations}

\section{Conflicts of interest}

The authors declare that they have no competing interests. 


\section{Availability of data and materials}

All data generated or analyzed during this study are included in this published article (and its additional file).

\section{Funding}

This work was supported by the National Natural Science Foundation of China (Grant No. 51772316, 81630046 and 81471659$)$

\section{Acknowledgements}

We thank prof. Jingxiang Chen for article modification.

\section{References}

1. Xiong F, Hu K, Yu H, Zhou L, Song L, Zhang Y, Shan X, Liu J, Gu N. Pharm Res. 2017;34:1683-92.

2. Ma W, Fu F, Zhu J, Huang R, Zhu Y, Liu Z, Wang J, Conti PS, Shi X, Chen K. Nanoscale. 2018;10:6113-24.

3. Cassidy M, Chan H, Ross B, Bhattacharya P, Marcus C. Nat Nanotechnol. 2013;8:363-8.

4. Wang S, Lin J, Wang Z, Zhou Z, Bai R, Lu N, Liu Y, Fu X, Jacobson O, Fan W. Adv Mater. 2017;29:1701013.

5. Schweiger C, Pietzonka C, Heverhagen J, Kissel T. Int J Pharm. 2011;408:130-7.

6. Carvalho A, Gonçalves M, Corvo M, Martins M. Magn Reson Ins. 2017;10:1178623 × 17722134.

7. Chen L, Xie J, Wu H, Li J, Wang Z, Song L, Zang F, Ma M, Gu N, Zhang Y, J. Nanomater., 2018, 2018.

8. Shen Z, Wu A, Chen X. Mol Pharmaceutics. 2017;14:1352-64.

9. Ma X, Gong A, Xiang L, Chen T, Gao Y, Liang X, Shen Z, Wu A. J Mater Chem B. 2013;1:3419-28.

10. Jenjob R, Kun N, Ghee J, Shen Z, Wu X, Cho S, Lee D, Yang S. Mater Sci Eng. 2016;61:659-64.

11. Jenjob R, Kun N, Ghee J, Shen Z, Wu X, Cho S, Lee D, Yang S. Mat Sci Eng C-Mater. 2016;61:659-64.

12. Hachani R, Lowdell M, Birchall M, Hervault A, Mertz D, Begin-C and Thanh N. Nanoscale. 2016;8:3278-87.

13. Liu Q, Song L, Chen S, Gao J, Zhao P, Du J. Biomaterials. 2017;114:23-33.

14. Wu A, Ou P, Zeng L. Nano. 2010;5:245-70.

15. Saeed M, Ren W, Wu A. Biomater Sci. 2018;6:708-25.

16. Wang H, Revia R, Wang K, Kant R, Mu Q, Gai Z, Hong K, Zhang M. Adv Mater. 2017;29:1605416.

17. Shen Z, Chen T, Ma X, Ren W, Zhou Z, Zhu G, Zhang A, Liu Y, Song J, Li Z. ACS Nano. 2017;11:10992-1004. 
18. Chen F, Bu W, Lu C, Chen G, Chen M, Shen X, Liu R, Shi J. J Nanosci Nanotechnol. 2011;11:1043843.

19. Gao Z, Ma T, Zhao E, Docter D, Yang W, Stauber R, Gao M. Small. 2016;12:556-76.

20. Ma D, Chen J, Luo Y, Wang H, Shi X. J Mater Chem B. 2017;5:7267-73.

21. Huang Y, Mao K, Zhang B, Zhao Y. Mat Sci Eng C-Mater. 2017;70:763-71.

22. Slobozhanyuk A, Poddubny A, Raaijmakers A, van Den Berg C, Kozachenko A, Dubrovina I, Melchakova I, Kivshar Y, Belov P. Adv Mater. 2016;28:1832-8.

23. Shen Z, Song J, Zhou Z, Yung B, Aronova M, Li Y, Dai Y, Fan W, Liu Y, Li Z. Adv Mater. 2018;30:1803163.

24. Chen L, Xie J, Wu H, Li J, Wang Z, Song L, Zang F, Ma M, Gu N, Zhang Y. J Nanomater. 2018. DOI:10.1155/2018/3743164.

25. Maraloiu V, Appaix F, Broisat A, Le Guellec D, Teodorescu V, Ghezzi C, van der Sanden B, Blanchin M. Nanomedicine. 2016;12:191-200.

26. Carron S, Bloemen M, Vander Elst L, Laurent S, Verbiest T, Parac-Vogt T. Chem Eur J. 2016;22:45217.

27. Czarniecki M, Pesapane F, Wood B, Choyke P, Turkbey B. Transl Androl Urol. 2018;7:453.

28. Lang J, Zhao X, Wang X, Zhao Y, Li Y, Zhao R, Cheng K, Li Y, Han X, Zheng X. ACS Nano. 2019;13:2176-89.

29. Shen L, Bao J, Wang D, Wang Y, Chen Z, Ren L, Zhou X, Ke X, Chen M, Yang A. Nanoscale. 2013;5:2133-41.

30. Ma X, Gong A, Chen B, Zheng J, Chen T, Shen Z, Wu A. Colloids Surf B. 2015;126:44-9.

31. Li J, Hu Y, Yang J, Sun W, Cai H, Wei P, Sun Y, Zhang G, Shi X, Shen M. J Mater Chem B. 2015;3:572030.

32. Ma Y, Huang J, Song S, Chen H, Zhang Z. Small. 2016;12:4936-54.

33. Luo Y, Yang J, Yan Y, Li J, Shen M, Zhang G, Mignani S, Shi X. Nanoscale. 2015;7:14538-46.

\section{Figures}

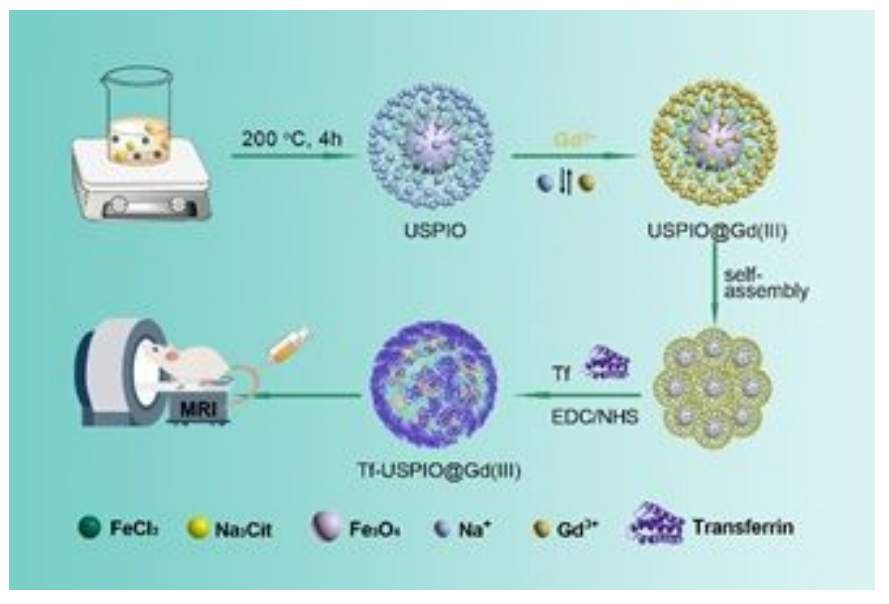


Figure 1

Schematic illustration of the preparation of TUG nanoprobes.

a
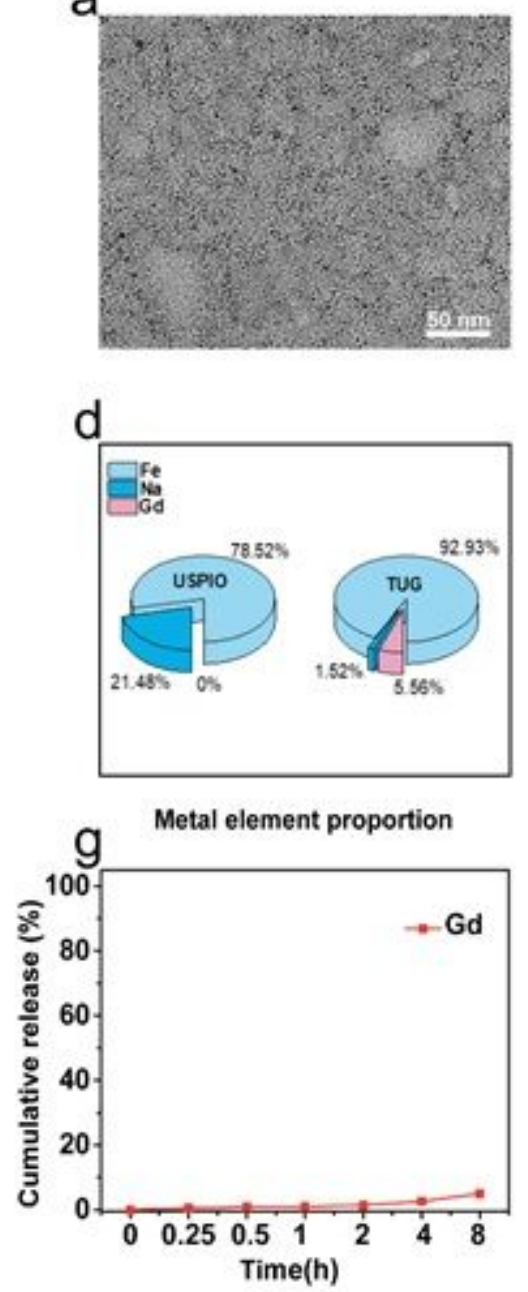

b

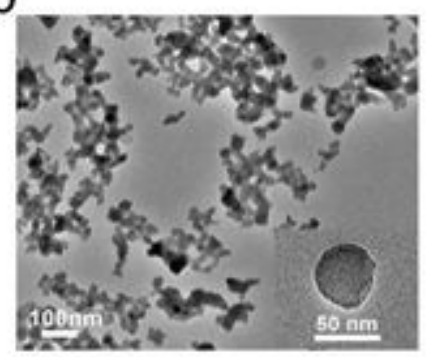

e

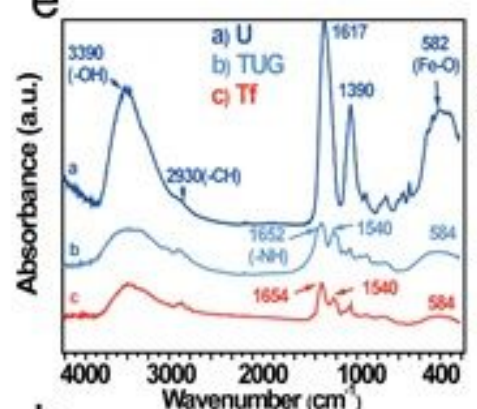

$\mathrm{h}$

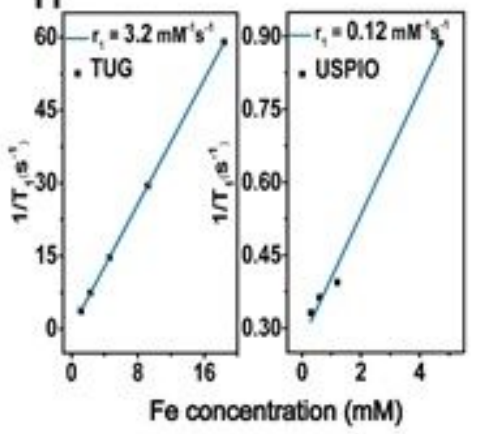

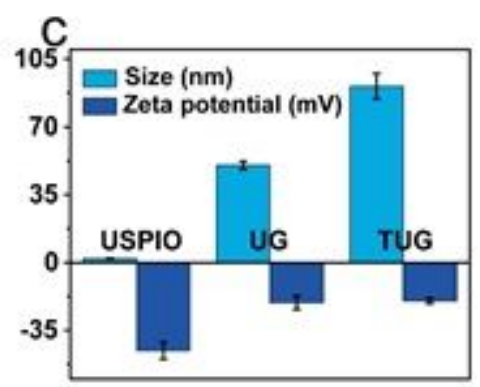
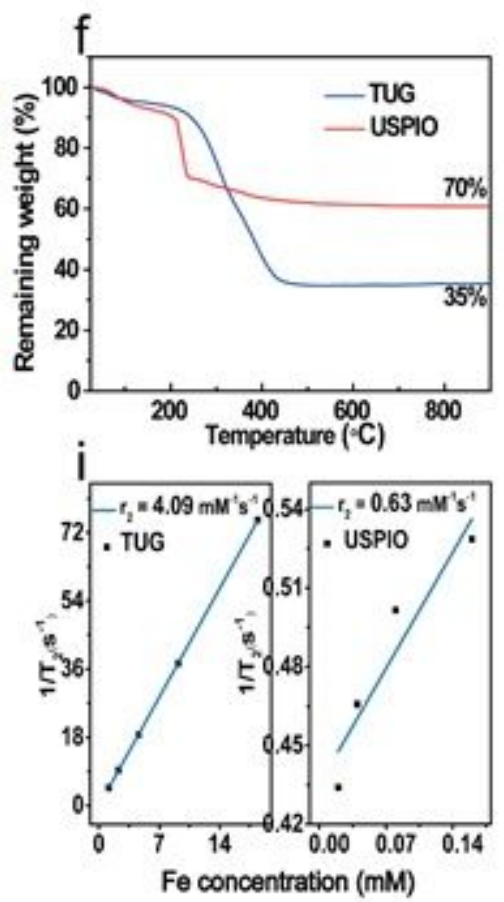

\section{Figure 2}

TEM images of (a) USPIO; (b) TUG with Fe/Gd ratio of 10 (inset shows an enlarged nanoparticle); (c) Hydrodynamic sizes and Zeta potentials of USPIO, UG, and TUG; (d) Metal element analysis of USPIO and TUG by ICP-OES; (e) FTIR spectra of TUG; (f) TGA curves of USPIO and TUG; (g) Gadolinium ion release curve of TUG; (h-i) Plot of 1/T1 and 1/T2 over Fe concentration of TUG and USPIO nanoparticles, the slope indicates the specific relaxivity $(r 1, r 2)$. 

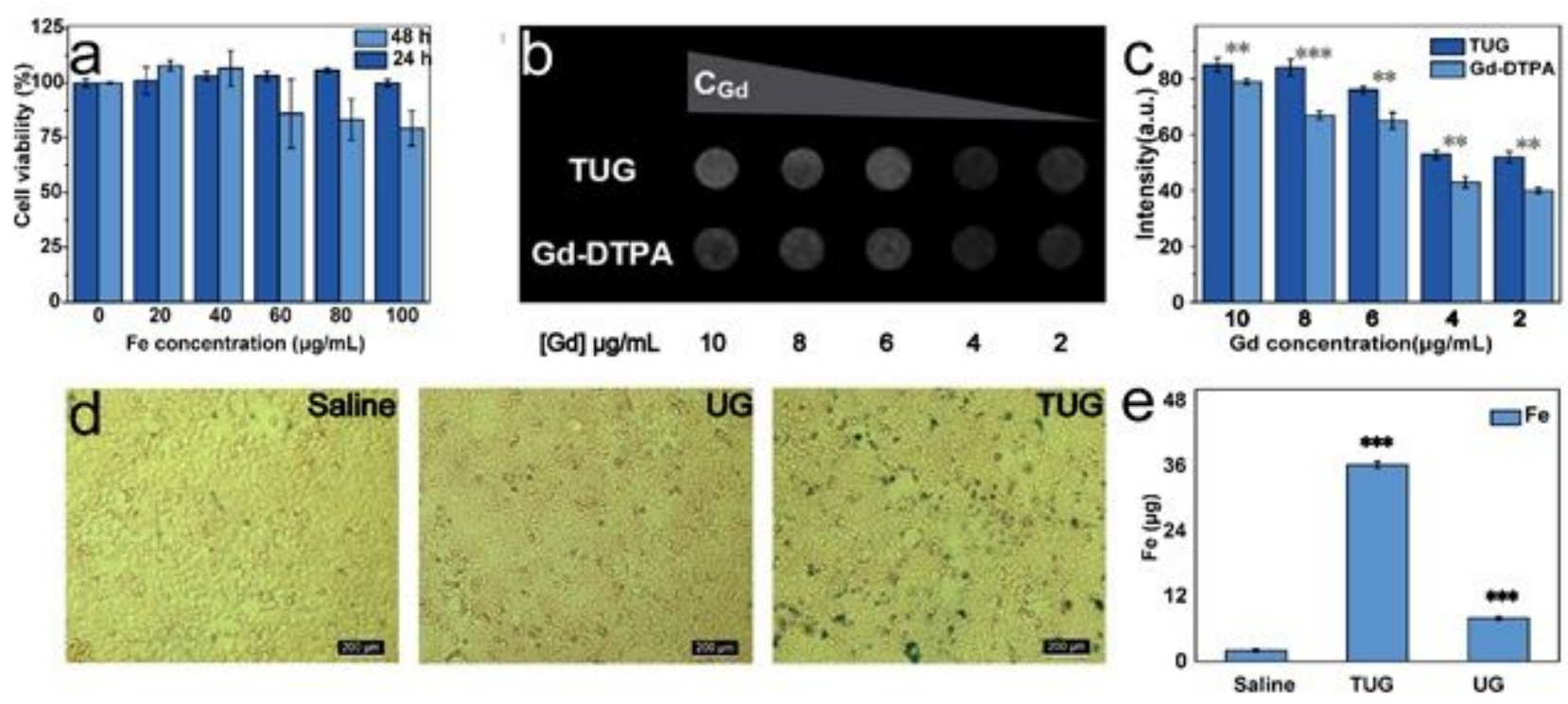

\section{Figure 3}

The viability of $4 \mathrm{~T} 1$ cells after coculture with TUG at different Fe concentrations for $24 \mathrm{~h}$ or $48 \mathrm{~h}$ measured by the CCK8 assay (a); In vitro T1-weighted MR images of 4T1 cells treated with the TUG and Gd-DTPA for $4 \mathrm{~h}$ (both $\mathrm{Gd}$ concentration 10, 8, 6, 4, $2 \mu \mathrm{g} / \mathrm{mL}$ ) (b); MR imaging signal values of $4 \mathrm{~T} 1 \mathrm{cells}$ after treatment with TUG and Gd-DTPA for $4 \mathrm{~h}$ (c); Prussian blue staining of 4 T1 cells after treated with the saline, UG, and TUG at the Fe concentration of $(10 \mu \mathrm{g} / \mathrm{mL})$, respectively for $4 \mathrm{~h}(\mathrm{~d})$. The cellular uptake of UG or TUG after treated with the TUG or UG nanoprobes for $4 \mathrm{~h}(10 \mu \mathrm{g} / \mathrm{mL})$. $4 \mathrm{~T} 1$ cells treated with saline were used as control (e). 
a

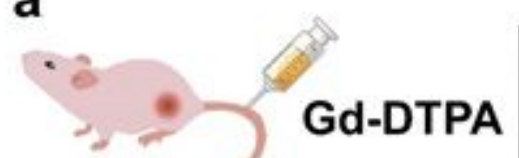

Xenografted tumor

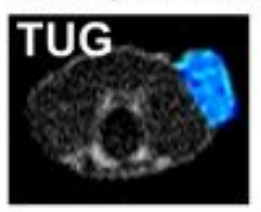

$\mathrm{Oh}$

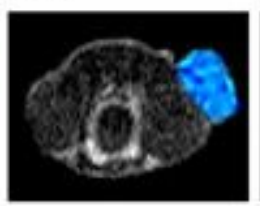

$2 \mathrm{~h}$

b

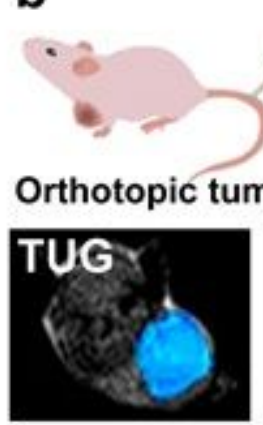

$\mathrm{Oh}$

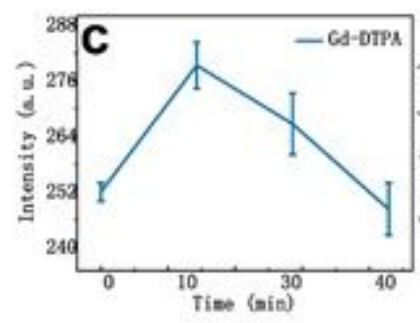

Gd-DTPA
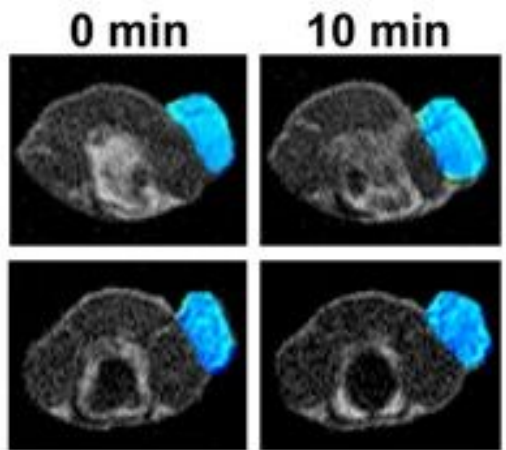

$4 \mathrm{~h}$
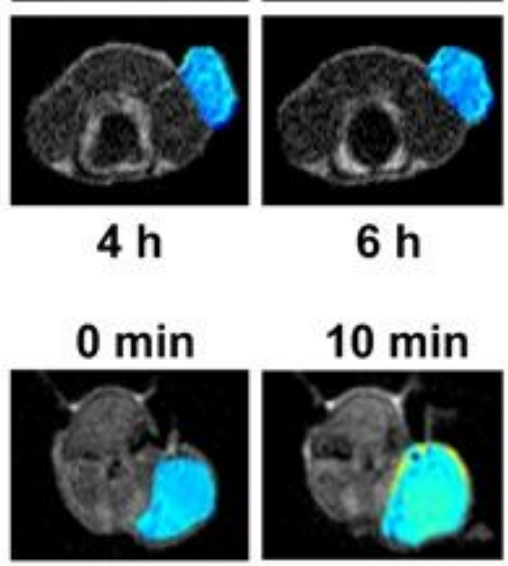

$6 \mathrm{~h}$
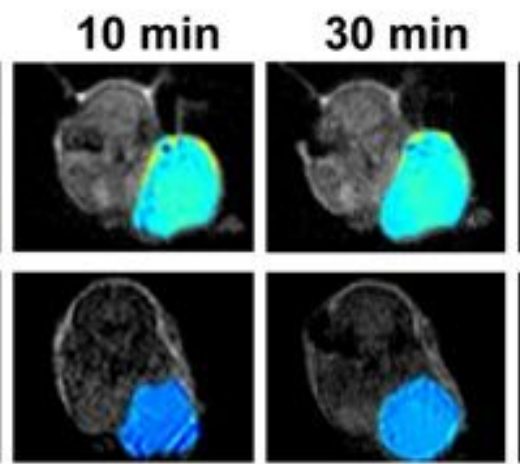

$4 \mathrm{~h}$

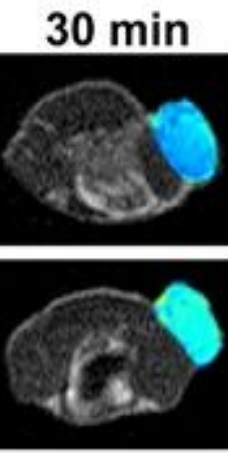

$8 \mathrm{~h}$

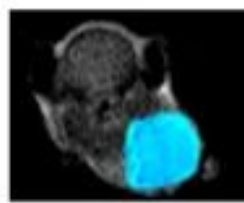

$2 \mathrm{~h}$

$6 \mathrm{~h}$

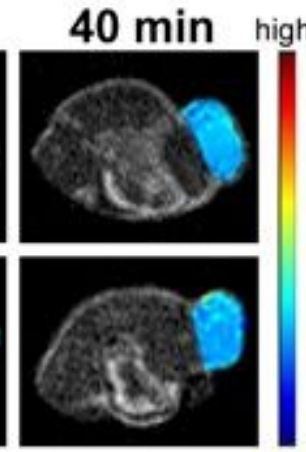

$10 \mathrm{~h}$ low

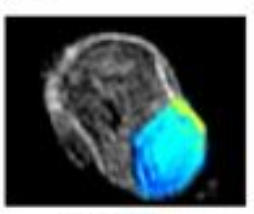

$0.5 \mathrm{~h}$
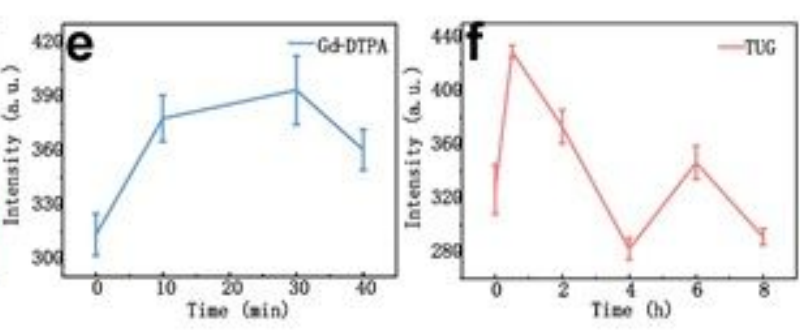

Figure 4

MR imaging performance of Gd-DTPA and TUG nanoprobes in vivo. The images are composed of a pseudocolor image of tumor area and MRI image of the same area. (a) MRI images of subcutaneous tumor in Balb/c nude mice after injection of TUG and Gd-DTPA; (b) MRI images of tumor in situ in Balb/c nude mice after injection of TUG and Gd-DTPA; (c) MR imaging signal value of subcutaneous tumor area after injection of Gd-DTPA in Balb/c nude mice; (d) MR imaging signal value of subcutaneous tumor area after injection of TUG in Balb/c nude mice; (e) MR imaging signal value of tumor in situ after injection of Gd-DTPA in Balb/c nude mice; (f) MR imaging signal value of tumor in situ after injection of TUG in Balb/c nude mice. 


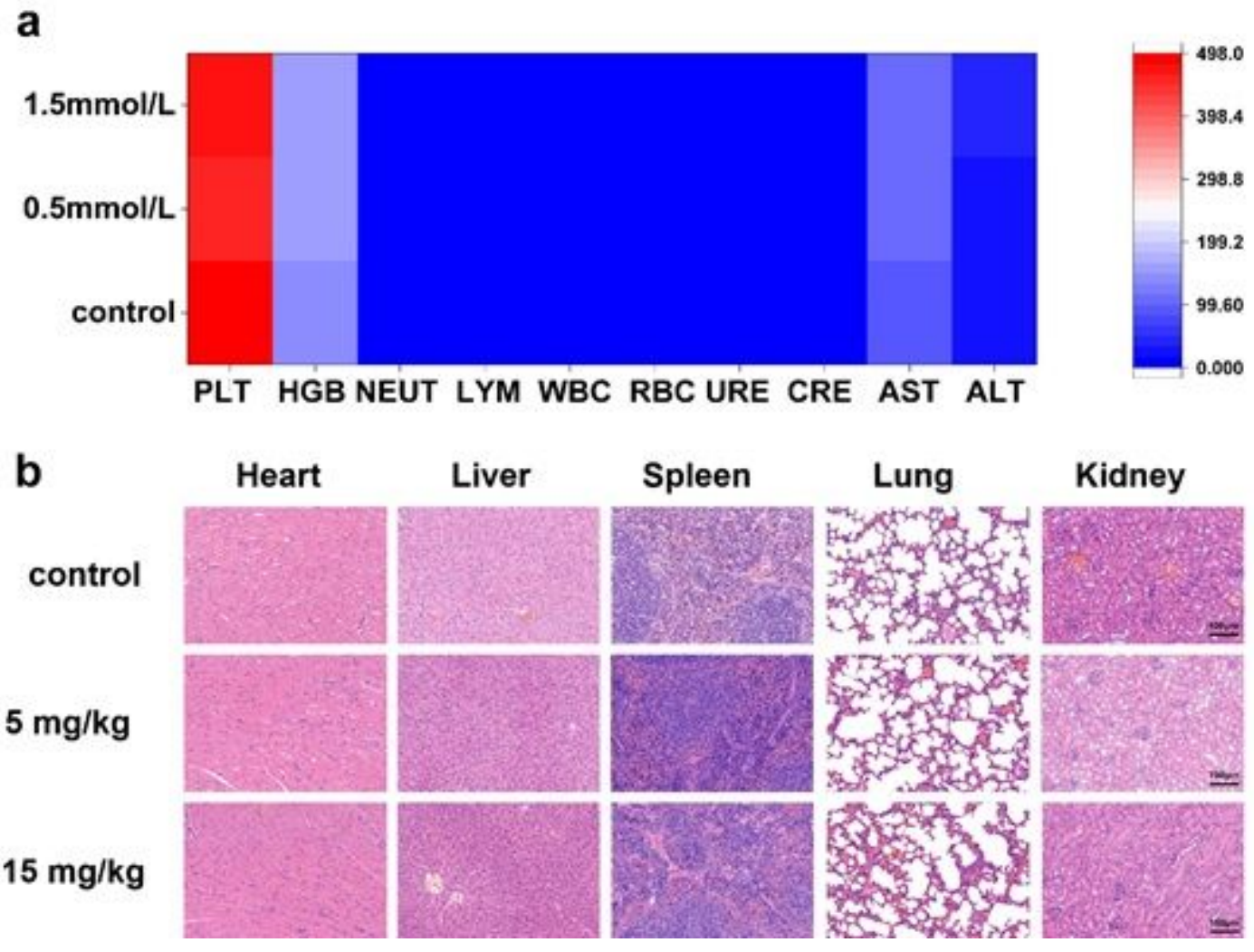

Figure 5

Biosafety experiments of TUG nanoprobes. (a) Blood analysis of mice after tail-vein injection of TUG nanoprobes $5 \mathrm{mg} / \mathrm{kg}(\mathrm{Fe})$. (b) H\&E-stained tissue slices after tail-vein injection of saline and TUG nanoprobes $5 \mathrm{mg} / \mathrm{kg}$ and $15 \mathrm{mg} / \mathrm{kg}$ (Fe). All tissue sections were stained on the 14th day after the injection of TUG.

\section{Supplementary Files}

This is a list of supplementary files associated with this preprint. Click to download.

- SI04201.docx 